\title{
Generalized binomial multiplicative cascade processes and asymmetrical multifractal distributions
}

\author{
Q. Cheng ${ }^{1,2}$ \\ ${ }^{1}$ State Key Lab of Geological Processes and Mineral Resources, China University of Geosciences, Beijing and Wuhan, China \\ ${ }^{2}$ Department of Geography, York University, Toronto, M3J1P3, Canada
}

Correspondence to: Q. Cheng (qiuming@yorku.ca)

Received: 29 September 2013 - Revised: 13 February 2014 - Accepted: 14 February 2014 - Published: 4 April 2014

\begin{abstract}
The concepts and models of multifractals have been employed in various fields in the geosciences to characterize singular fields caused by nonlinear geoprocesses. Several indices involved in multifractal models, i.e., asymmetry, multifractality, and range of singularity, are commonly used to characterize nonlinear properties of multifractal fields. An understanding of how these indices are related to the processes involved in the generation of multifractal fields is essential for multifractal modeling. In this paper, a fiveparameter binomial multiplicative cascade model is proposed based on the anisotropic partition processes. Each partition divides the unit set (1-D length or 2-D area) into $h$ equal subsets (segments or subareas) and $m_{1}$ of them receive $d_{1}(>0)$ and $m_{2}$ receive $d_{2}(>0)$ proportion of the mass in the previous subset, respectively, where $m_{1}+m_{2} \leq h$. The model is demonstrated via several examples published in the literature with asymmetrical fractal dimension spectra. This model demonstrates the various properties of asymmetrical multifractal distributions and multifractal indices with explicit functions, thus providing insight into and an understanding of the properties of asymmetrical binomial multifractal distributions.
\end{abstract}

\section{Introduction}

Singular physical, chemical and biological processes can result in anomalous energy release, mass accumulation or matter concentration, which are all generally confined to narrow intervals in space or time (Cheng, 2007a). The end products of these nonlinear processes can all be modeled as fractals or multifractals. Singularity is a property of nonlinear natural processes, examples of which include but are not lim- ited to cloud formation (Schertzer and Lovejoy, 1987), rainfall (Veneziano, 2002), hurricanes (Sornette, 2004), flooding (Malamud et al., 1996; Cheng, 2008; Cheng et al., 2009), landslides (Malamud et al., 2004), forest fires (Malamud et al., 1996), earthquakes (Turcotte, 1997), mineral deposits (Agterberg, 1995; Cheng et al., 1994; Cheng and Agterberg, 2009), and solar wind turbulence (Macek, 2007). Multifractal modeling involves quantification of multiscale singularities and various types of properties associated with distribution of singularities. In addition to the commonly used indices such as multifractality and asymmetry of multifractal distributions, other types of properties of multifractal distribution such as spatiotemporal stationarity (Lombardo et al., 2012, 2013), lacunarity (Mandelbrot, 1983; Plotnick et al., 1993; Cheng, 1997b) and variability (autocorrelation and variogram) (Cheng and Agterberg, 1996) are also essential for characterization and application of multifractal distributions. Common questions in the application of multifractal model to real-world problems are whether the phenomenon depicts multifractality and the significance of the multifractality in comparison with monofractals or nonfractals. Asymmetry of the multifractal distribution is another interesting property of the multifractal distribution. Answering these questions is necessary not only to understand the phenomenon but also to choose methodologies for implementation (Cheng and Agterberg, 1996; Cheng, 1997a, 1999b). In the current paper, a five-parameter binomial multiplicative cascade process is introduced to illustrate the links between commonly used multifractal indices and the processes involved in generation of the asymmetrical binomial multifractal distribution.

To introduce the new model, we first present selected mathematical notations and concepts of the multiplicative 
cascade process, a simple multifractal model and the associated singularities. Several formalisms exist for representing multifractals, e.g., deterministic and stochastic models. A complete review of various multifractal models is beyond of the scope of this paper, and therefore, in this work, we present only the relevant mathematical notation for the multifractal model based on partition functions (Halsey et al., 1986). The main reason for choosing this model for discussion is that, firstly, this partition functions-based model is well known in most fields where multifractal modeling is originally applied not only for 1-D time series but also for 2-D fields and, secondly, the similar discussion can be easily extended to describe other multifractal models. For convenience without loss of generality we will use a 1-D problem for discussion. We assume a measure $\mu$ defined in a small segment within a finite 1-D set T (e.g., a line segment), and its value with a linear measuring size $\varepsilon$ satisfies

$\mu(\varepsilon) \propto \varepsilon^{\alpha}$,

where $\alpha$ stands for "proportional to" and $\alpha$ is the singularity index (also known as the coarse Hölder exponent), and $\mu$ is a function of scale $\varepsilon$ that possesses an isotropic scale invariance property such that the following ratio of logarithmic transformations of $\mu$ and $\varepsilon$ yields a scale-independent index when $\varepsilon$ approaches zero:

$\alpha \propto \frac{\log [\mu(\varepsilon)]}{\log \varepsilon}$.

The values of $\alpha$ usually vary within a finite interval $\left[\alpha_{\min }\right.$, $\left.\alpha_{\max }\right]$ for deterministic multifractals, but for other models (e.g., an entire family of the universal multifractal models, Schertzer and Lovejoy, 1987, that includes both the $\beta$ model, Frisch et al., 1978, and the log-normal model, Yaglom, 1966) the bound of singularity might be infinite (Lovejoy and Schertzer, 2007). For certain models, the value of singularity can be negative, and the fractal dimension can be negative as well (Cheng, 1997a). According to the distribution of the value of $\alpha$, the entire set ( $\mathrm{T}$ ) can be classified into subsets or fractals, each of which possesses a different singularity value $\alpha_{i}$ and, accordingly, different fractal dimensions $\left(f\left(\alpha_{i}\right) \leq 1\right)$; this is the reason why the field of $\mu$ is described by the term "multifractality". The fractal dimension function $f(\alpha)$ and the singularity $\alpha$ can be estimated using various multifractal methods. Using the terminologies pertaining to the multifractal model based on the partition function (Halsey et al., 1986), the three functions - the mass exponent function or Renyi exponent $\tau(q)$, the coarse Hölder exponent $\alpha(q)$, and the fractal spectrum function $f(\alpha)$ - involved in the multifractal model based on the partition functions have the following relationships (Halsey et al., 1986):

$$
\begin{aligned}
& \sum[\mu(\varepsilon)]^{q} \propto \varepsilon^{\tau(q),} \\
& a(q)=\tau^{\prime}(q), \\
& f(a)=a q-\tau(q),
\end{aligned}
$$

where $q$ is the order of moment, and the summation in the first equation is applied for all segments of equal size $\varepsilon$ with positive measure $\mu$. If we assume that the number $N_{\alpha}(\varepsilon)$ of segments with size $\varepsilon$ covers the entire subset bearing the singularity $\alpha$, the fractal dimensions $f(\alpha)$ of this subset are related by

$N_{a}(\varepsilon) \propto \varepsilon^{-f(a)}$.

The total measure of the subset can be expressed as

$N_{a}(\varepsilon) \mu_{a}(\varepsilon) \propto \varepsilon^{-f(a)+\alpha}$.

According to Eqs. (1)-(5) one can derive the following properties of $\tau(q), \alpha(q)$, and $f(\alpha)$ (Cheng, 1999a):

$$
\begin{aligned}
& a \geq f(a), \\
& \frac{\partial \alpha}{\partial q}=\frac{\partial^{2} \tau(q)}{\partial q^{2}} \leq 0, \\
& \frac{\partial^{2} f(\alpha)}{\partial \alpha^{2}}=\frac{1}{\frac{\partial^{2} \tau}{\partial q^{2}}}<0 .
\end{aligned}
$$

Equation (6a) holds true because the total measure of the subset is less than the total measure of the entire set. If Eq. (6a) is not true, then the total measure of the subset Eq. (5) will approach infinity when $\varepsilon \rightarrow 0$. The proof of Eqs. (6b) and (6c) can be found in Cheng (1999a). The properties of Eqs. (6b) and (6c) ensure that the functions $\tau(q)$ and $f(\alpha)$ are concave functions and $\alpha(q)$ is a monotonic decreasing function. The frequency distribution of the measure characterized by singularity $\alpha$ in the entire set can be described by the fractal dimension spectrum function $f(\alpha)$. From this formulation, we can extract the following properties. When $q=0$ and $\alpha(0), f(\alpha(0))=-\tau(0)$, reaching the maximum value of $f(\alpha)$, which corresponds to the box-counting fractal dimension. If the measure covers the entire set, then the boxcounting dimension equals 1 , otherwise it is less than 1 . When $q=1$ and $\alpha(1), f(\alpha(1))=\alpha(1)-\tau(1)$. If the first moment $\sum \mu(\varepsilon)=$ constant, then $\tau(1)=0$ and $f(\alpha(1))=$ $\alpha(1)$, corresponding to conservative measure, otherwise, if $\tau(1) \neq 0$, the measure is not conservative. The maximum value $f(\alpha(0))$ or the box-counting dimension of the support of $\mu$ at $\alpha(0)$ implies that the majority of the segments have a measure characterized by $\alpha \approx \alpha(0)$, whereas segments with values $\alpha>\alpha(0)$ or $\alpha<\alpha(0)$ are more irregular and have fractal dimensions $f(\alpha)<f(\alpha(0))$.

In addition to the complete fractal dimension spectrum, functions of singularity or generalized dimension functions, functions of order of moment, and several multifractal indices are commonly used for characterization of multifractality and asymmetry of multifractal fields. For example, the curvature of functions $\tau(q)$, and $f(\alpha)$ or range of value $\alpha(q)$ can all be used for measuring multifractality (Cheng et al., 1994; Cheng and Agterberg, 1996). Halsey et al. (1986) suggested the use of the range of singularity $\Delta \alpha=\alpha_{\max }-\alpha_{\min }$ 
to measure the multifractality, and Schertzer and Lovejoy (1987) proposed a universal multifractality model that involves three parameters, one of the indices based on the curvature of co-dimension function for measuring multifractality. Cheng and Agterberg (1996) proposed an index for measuring multifractality based on the curvature of mass exponent function or Renyi exponent $\tau(q)$ at $q=1, \Delta \tau=$ $-[\tau(2)-2 \tau(1)+\tau(0)]$ or the second derivative $-\tau^{\prime \prime}(1)$, among others. In terms of asymmetry indices, there are several suggestions. For example, Shimizu et al. (2002) suggested two ways to measure the asymmetry of multifractal spectra: when a quadratic function fitted by the least square method to the fractal dimension spectra around the position of their maxima at $\alpha(0)$, a parameter $(B)$ involved in the quadratic function can be used to quantify the asymmetry of the quadratic function. Parameter $B$ serves as an asymmetry parameter, which is zero for symmetric shapes and positive or negative for a left- or right-skewed shape, respectively. In their paper, the alternative index or the unsigned ratio $R=\left|\frac{\text { left slope }}{\text { right slope }}\right|$, which is equivalent to $R=(\alpha(0)-$ $\left.\alpha_{\min }\right) /\left(\alpha_{\max }-\alpha(0)\right)$, was proposed as an asymmetry index with $R>1$ indicating that the entire spectrum is bent to the left (left-skewed) and $R<1$ indicating that the curve is bent to the right (right-skewed). These authors suggested the use of a vector containing three indices $(\alpha(0), R, \Delta \alpha)$ as a measure of the complexity of multifractals. Other similar asymmetry indices were suggested and applied by Xie and Bao (2004). In addition to the multifractality and asymmetry indices, other characteristic values were also used, i.e., the boxcounting dimension $f(\alpha(0))=f_{\max }$ and the dimensions of extreme values at two ends of spectra $f\left(\alpha_{\max }\right)$ and $f\left(\alpha_{\min }\right)$. It is worthwhile to mention that the estimation of Eq. (3) can be affected by various factors including presence of finite size effect (FSE), additive white or color noise, shortterm memory or periodicities in multifractal signals. Some indices such as $\Delta \alpha$ may be meaningless if the whole range of singularity functions cannot be accurately estimated. These indices have to be used with caution (Grech and Pamuła, 2013). An understanding of the physical meaning of these indices is essential for applications of these indices in geosciences for interpretation of geoprocesses corresponding to the formation of multifractal distributions. In the current paper, a five-parameter binomial multiplicative cascade process is introduced to illustrate the links between these indices and the processes involved in generation of the asymmetrical binomial multifractal distribution. In the next section, we will show how binomial multiplicative cascade processes can be used to derive explicit functions of these indices and demonstrate how these indices are related to the multiplicative cascade processes in the generation of multifractal distributions.

\section{Multiplicative cascade processes and multifractal distributions}

The theories and concepts of multiplicative cascade processes (MCP) play a fundamental role in quantifying turbulent intermittency and other nonlinear processes (Schertzer and Lovejoy, 1985, Schertzer et al,. 1997). Therefore, MCP has been extensively discussed in the literature (e.g., Gupta and Waymire, 1993; Over and Gupta, 1996; Menabde and Sivapalan, 2000; Serinaldi, 2010). The model of de Wijs is a simple two-dimensional multiplicative cascade model (de Wijs, 1951; Agterberg, 2001, 2007a) described in terms of multiplicative canonical cascade processes (Lovejoy and Schertzer, 2007). Other modified models exist, e.g., a cascade model with functional redistribution rate (Agterberg, 2007b), a 2-D cascade model with anisotropic partition processes (Cheng, 2005), a 2-D cascade model with variable and conditional dependence partition processes (Cheng, 2012), a generalized two-parameter binomial multiplicative model that was proposed by Koscielny-Bunde et al. (2006) and applied for describing multifractal spectra of runoff time series, and a three-parameter binomial multifractal model that was proposed by Macek (2007) and applied to characterize solar wind turbulence data based on a generalized twoscale weighted cantor set for characterizing asymmetrical multifractal distribution. Macek's model has been successfully applied for modeling solar minimum and maximum values (Macek and Szczepaniak, 2008; Macek and Wawrzaszek, 2011). Macek's model generates an asymmetrical multifractal distribution and can be used to fit asymmetrical multifractal distributions. In this paper, a five-parameter binomial multiplicative cascade model which gives explicit forms of fundamental multifractal indices characterizing asymmetrical multifractal distribution of real-world data, is proposed. The new model gives explicit relationships between the multifractal indices and the parameters involved in the binomial multiplicative cascade processes. Moreover, the new model is able to generate an asymmetrical multifractal distribution with nonzero fractal dimensions of the extreme values at two ends of the spectrum, i.e., $f\left(\alpha_{\max }\right) \neq 0$ and $f\left(\alpha_{\min }\right) \neq 0$. For simplicity without loss of generality, we first introduce a 1D normal binomial multiplicative cascade model that leads to a symmetrical multifractal distribution and subsequently extend it to a more general binomial model that leads to an asymmetrical multifractal distribution. Finally, several examples are used to demonstrate the use of the new model. The similar discussions can be extended to 2-D multifractal models.

\subsection{Normal binomial multiplicative cascade model and symmetrical multifractal distribution}

The simple version of de Wijs' cascade model involves the partitioning of each unit segment into two subsegments of equal size. The concentration value $(\rho)$ of a quantity in the 
unit segment can be written as $d \cdot \rho$ for one half and $(1-d) \cdot \rho$ for the other half $(0<d<1)$ such that the total mass is preserved. The coefficient of dispersion $d$ is independent of segment size. At the beginning of the process, the value of $\rho$ for the first segment can be set equal to unity. If $d>0.5$, the maximum element concentration value after $n$ subdivisions is $\mu=d^{n}$, and the minimum value is $\mu=(1-d)^{n}$; if $d<0.5$, the maximum and minimum concentrations are switched. The general value of the concentration after $n$ subdivisions can be represented as $\mu=d^{k}(1-d)^{n-k}$, where $0 \leq k \leq n$. The number of segments with this value is $\left(\begin{array}{l}n \\ k\end{array}\right)$. In a random cascade, larger and smaller values are assigned to segments using a discrete random variable. The frequency distribution of the element concentrations at any stage of this process is referred to a "logbinomial" because the logarithmically transformed concentration values satisfy a binomial distribution. The logbinomial converges to a log-normal distribution, although its upper and lower value tails remain weaker than those of the log-normal (Agterberg, 2007a). Due to its property of self-similarity, the model of de Wijs was recognized as a multifractal by Mandelbrot (1989), who adopted this approach for applications to the elements in Earth's crust.

Let $k / n=\xi$, the value of $\mu(\xi)=\left[d^{\xi}(1-d)^{1-\xi}\right]^{n}$ and the number of cells with size $\varepsilon_{n}=(1 / 2)^{n} ; \mu(\xi)$ is then $N\left(\varepsilon_{n}\right)=$ $\left(\begin{array}{l}n \\ k\end{array}\right)$. Therefore, the multifractal patterns generated by this cascade process contain many local maxima and minima, with singularities expressed as follows (Feder, 1988; Cheng, 2005):

$$
\begin{aligned}
\alpha & =-\frac{\xi \ln (d)+(1-\xi) \ln (1-d)}{\ln 2}, \\
f(\alpha) & =-\frac{\xi \ln \xi+(1-\xi) \ln (1-\xi)}{\ln 2},
\end{aligned}
$$

where $\xi$ is a value with $0 \leq \xi \leq 1$. The fractal dimension spectrum $f(\alpha)$ characterizes the dimension of the distribution of singularity.

To discuss the property of multifractals generated by multiplicative cascade processes, we use moment notation to derive the functions involved in the general multifractal modeling. The partition function at various scales can be defined as

$$
\begin{aligned}
\chi_{q}\left(\varepsilon_{n}\right) & =\sum_{k=0}^{n} \mu_{k}\left(\varepsilon_{n}\right)^{q}\left(\begin{array}{l}
n \\
k
\end{array}\right)=\sum_{k=0}^{n}\left[d^{k}(1-d)^{n-k}\right]^{q}\left(\begin{array}{l}
n \\
k
\end{array}\right) \\
& =\left[d^{q}+(1-d)^{q}\right]^{n} .
\end{aligned}
$$

Therefore, it can be rewritten as

$\chi_{q}\left(\varepsilon_{n}\right)=\varepsilon_{n}^{-\frac{\log \left[d^{q}+(1-d)^{q}\right]}{\log 2}}=\varepsilon_{n}^{\tau(q)}$,

where the mass function is

$\tau(q)=-\frac{\log \left[d^{q}+(1-d)^{q}\right]}{\log 2}$ and according to the relationship between the mass functions and singularity functions, the singularity index and the fractal dimension spectrum can be derived as follows:

$$
\begin{aligned}
a(q) & =\frac{\partial \tau(q)}{\partial q}=-\frac{\log d d^{q}+\log (1-d)(1-d)^{q}}{\log 2\left[d^{q}+(1-d)^{q}\right]} \\
& =-\frac{\xi \log d+(1-\xi) \log (1-d)}{\log 2}
\end{aligned}
$$

where we set

$$
\xi=\frac{d^{q}}{d^{q}+(1-d)^{q}}
$$

and

$$
\begin{aligned}
f(a) & =a(q) q-\tau(q)=-\frac{\xi \log d^{q}+(1-\xi) \log (1-d)^{q}}{\log 2} \\
& +\frac{\log \left[d^{q}+\left(1-d^{q}\right)\right]}{\log 2} \\
& =-\frac{\xi \log \xi+(1-\xi) \log (1-\xi)}{\log 2} .
\end{aligned}
$$

Equations (11) and (13) are indeed the same as Eqs. (7a) and $(7 b)$. The following relation can be derived from Eqs. (10)-(12)

$$
\frac{\partial \alpha}{\partial q}=\frac{\partial^{2} \tau}{\partial q^{2}}=-\frac{d^{q}(1-d)^{q}\left[\log \left(\frac{d}{1-d}\right)\right]^{2}}{\log 2\left[d^{q}+(1-d)^{q}\right]^{2}} \leq 0 .
$$

This relation shows that the function $\alpha(q)$ is a monotonic decreasing function and $\tau(q)$ a concave function. Similarly, according to Eq. (6c) the function $f(\alpha)$ is also a concave function. For the monotonic function of $\alpha(q)$ and concave functions of $\tau(q)$ and $f(\alpha)$, the following multifractal indices introduced in the Introduction are meaningful and can be derived according to Eqs. (10)-(13) as follows:

$$
\begin{aligned}
\alpha_{\max } & =\alpha(-\infty)=-\frac{\log (d)}{\log 2}, \alpha_{\min }=\alpha(\infty)=-\frac{\log (1-d)}{\log 2}, \\
\alpha(0) & =-\frac{\log [d(1-d)]}{2 \log 2}, \\
\Delta \alpha & =\alpha_{\max }-\alpha_{\min }=\log \frac{d}{(1-d)} / \log 2, \\
\Delta \alpha_{R} & =\alpha(\infty)-\alpha(0)=-\frac{1}{2 \log 2} \log \frac{d}{1-d}, \\
\Delta \alpha_{L} & =\alpha(0)-\alpha(-\infty)=-\frac{1}{2 \log 2} \log \frac{d}{1-d} \\
R & =\frac{\Delta \alpha_{l}}{\Delta \alpha_{r}}=1, \\
\Delta \tau & =-\tau^{\prime \prime}(1)=\frac{d(1-d)}{\log 2}\left[\log \left(\frac{d}{1-d}\right)\right]^{2} .
\end{aligned}
$$

These relations show that both multifractality indices $\Delta \alpha$ and $\Delta \tau$ are related to the choice of $d$. As the value $d$ approaches 
0.5 , the values of these indices reduce to zero, and if $d=0.5$, then $\Delta \alpha=0$ and $\Delta \tau=0$. According to the fractal dimension, the sets with maximum and minimum singularity have dimensions $f\left(\alpha_{\min }\right)=f\left(\alpha_{\max }\right)=0$, and the set with $\alpha(0)$ has the maximum dimension $f(\alpha(0))=1$.

To further illustrate the functions of $f(\alpha)$ and $\alpha(q)$, we draw the curves of these functions with various moments $q$ and dispersion values of $d$. For example, Fig. 1 shows the results with $d=0.1,0.3$, and 0.45 and various ranges of $q$. From Eqs. (11) and (13) as well as Fig. 1, we see that the distribution of a spectrum function is symmetrical and has the maximum value of $f(\alpha)=1$ at the middle range of the $\alpha(0)$ value, which corresponds to the value $\xi=0.5$ and $q=0$. This result indicates that the maximum dimension is 1 (1-D problem). From the curves in Fig. 1, we also can observe that as the dispersion $d$ increases from $0.1,0.3$ to 0.45 , the range of the $\alpha$ value decreases and the curve $\alpha(0)$ moves towards the left. From the distribution of the $\alpha$ function, we can observe that the range of $\alpha$ value increases with the decrease in the $d$ values $(d<0.5)$, but the range of the moment $q$ value decreases. Accordingly, the multifractality indices associated with these distributions are calculated as $\Delta \alpha=3.17,1.22$, and 0.29 , and $\Delta \tau=0.272,0.094$, and 0.006 , respectively. Each of the two sets of values characterizes the decreasing level of multifractality of the three multifractal distributions. These results also show that for a multifractal field with a large singularity and a large dispersion rate the available range of moments is usually small, which is a key consideration in estimation of multifractal distributions using moment methods. When multifractal models are applied to the real-world data it may encounter a problem for estimating the full range of singularity, especially for the two extreme large and small singularities due to limited resolution or finite size of data. There have been some methods for edge effect correction such as the effect-correction method proposed by Agterberg and Cheng (1996) and the glidingbox method (Cheng, 1999b). The problem of finite size effects (FSE) is currently under intensive debate (Grech and Pamuła, 2012, 2013). Therefore, some of the indices that involve the values of extreme singularities and fractal dimensions at two ends, such as $f\left(\alpha_{\max }\right)$ and $f\left(\alpha_{\min }\right)$, and $\alpha_{\max }$ and $\alpha_{\min }$, may involve large uncertainties and they have to be used with caution. From this point of view, the multifractality indices based on curvatures of the functions, such as $\Delta \tau$, may be superior to others, such as $\Delta \alpha$, based on the full range of singularity. The former can be accurately estimated by means of low order moments such as $\tau(2), \tau(1)$, and $\tau(0)$. Other factors affecting the accuracy of estimates of the multifractal indices include but are not limited to the noise or trend mixed with the data. Several suggestions such as filtering raw data to reduce the mixing effects of raw data in multifractal modeling have been proposed for solving these types of problems (Cheng, 2007b). Other frequently used techniques include the multifractal, detrended fluctuation analysis (MFDFA) (Kantelhardt et al., 2002), which enables one to elimi-

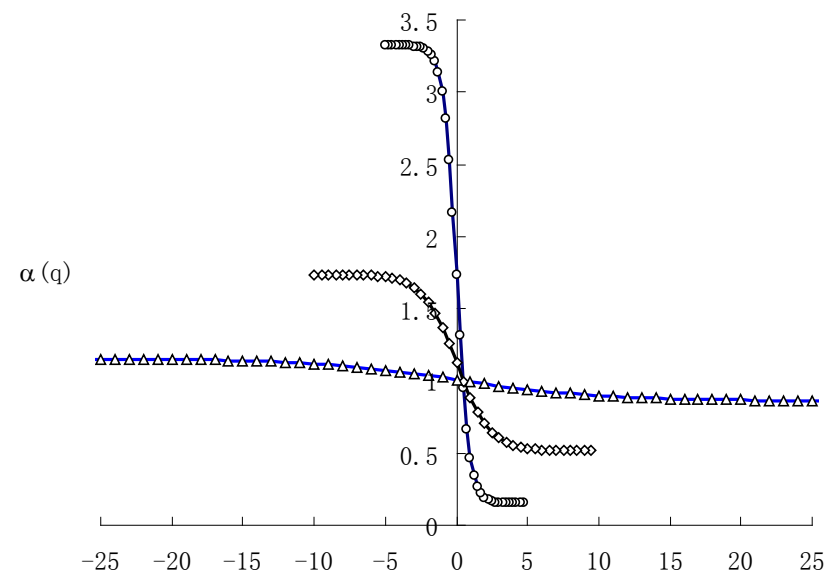

(a)

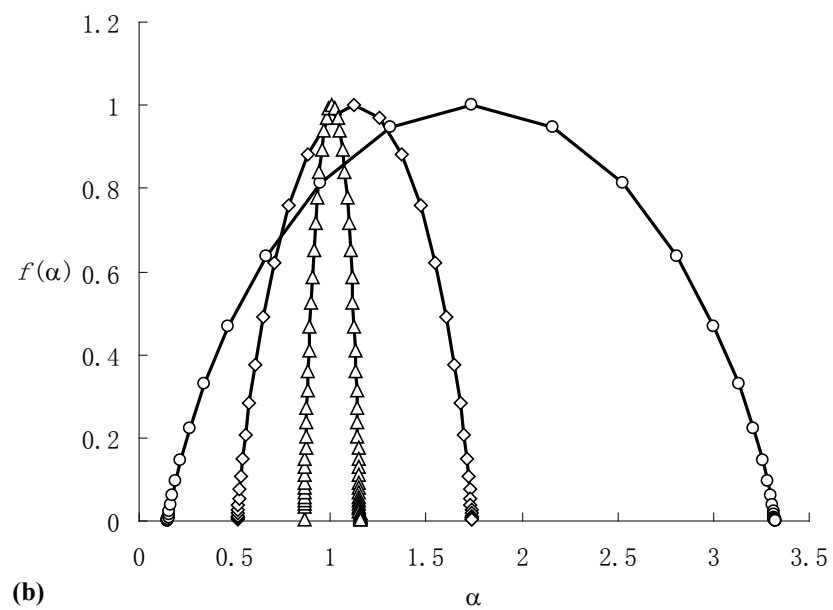

Fig. 1. (a) Singularity functions $\alpha(q)$ obtained from the normal de Wijs model with $d=0.1$ (triangles), 0.3 (diamonds) and 0.45 (dots), respectively. Value ranges of $q$ are different for models with different values of $d$. (b) The results of spectra functions $f(\alpha)$ for different values of $d$.

nate polynomial trends in time series data. More information about the MF-DFA and comparisons with other multifractal methods can be found in Kantelhardt et al. (2002). MF-DFA has also been extended to model 2-D sequence data (Rosas et al., 2002; Gu and Zhou, 2006; Telesca et al., 2007; Telesca and Lovallo, 2011).

\subsection{General binomial asymmetrical multiplicative cascade processes and asymmetrical multifractal distribution}

The multiplicative cascade processes introduced in the previous section involve symmetrical partition processes that generate multifractal distributions with symmetrical properties. In this section, we introduce an asymmetrical cascade process that generates asymmetrical multifractal distributions. Each partition divides the unit length into $h$ equal segments 
and $m_{1}$ of them receive $d_{1}(>0)$ and $m_{2}$ receives $d_{2}(>0)$ proportion of the mass in the previous segment, respectively, where $m_{1}+m_{2} \leq h$. For a closed system with preservation of unit measure, $d_{1}+d_{2}=1$. Otherwise, $d_{1}+d_{2}<1$ or $d_{1}+d_{2}>1$, corresponding to a loss or gain of mass during the cascade processes, respectively. At the $n$th partition, the segment length will be $\varepsilon_{n}=(1 / h)^{n}$, the segments are subject to $k$ times the segments with measure $d_{1} / m_{1}$ and $n-k$ times the segments with measure $d_{2} / m_{2}$, and thus the measures of these segments are $\mu_{\kappa}=\left(d_{1} / m_{1}\right)^{k}\left(d_{2} / m_{2}\right)^{n-k}$. Therefore, the numbers of segments with $\mu_{k}$ will be $N_{k}=$ $m_{1}^{k} m_{2}^{n-k}\left(\begin{array}{l}n \\ k\end{array}\right)$, and the partition function can be expressed as

$$
\begin{aligned}
\chi_{q}\left(\varepsilon_{n}\right) & =\sum_{k=0}^{n} \mu_{k}\left(\varepsilon_{n}\right)^{q} m_{1}^{k} m_{2}^{n-k}\left(\begin{array}{l}
n \\
k
\end{array}\right) \\
& =\sum_{k=0}^{n}\left[\left(d_{1} / m_{1}\right)^{k}\left(d_{2} / m_{2}\right)^{n-k}\right]^{q} m_{1}^{k} m_{2}^{n-k}\left(\begin{array}{l}
n \\
k
\end{array}\right) \\
& =\left[m_{1}^{1-q} d_{1}^{q}+m_{2}^{1-q} d_{2}^{q}\right]^{n} .
\end{aligned}
$$

From this partition function, one can derive the mass function as

$$
\tau(q)=-\frac{\log \left[m_{1}^{1-q} d_{1}^{q}+m_{2}^{1-q} d_{2}^{q}\right]}{\log h}
$$

and further derive the singularity index function $\alpha(q)$ as

$\alpha(q)=-\frac{\xi \log \left(d_{1} / m_{1}\right)+(1-\xi) \log \left(d_{2} / m_{2}\right)}{\log h}$,

where

$\xi=\frac{m_{1}^{1-q} d_{1}^{q}}{m_{1}^{1-q} d_{1}^{q}+m_{2}^{1-q} d_{2}^{q}}$.

From Eqs. (15) and (16), we obtain the spectrum function as

$f(\alpha)=\alpha(q) q-\tau(q)=-\frac{\xi \log \left(\frac{\xi}{m_{1}}\right)+(1-\xi) \log \left(\frac{1-\xi}{m_{2}}\right)}{\log h}$.

The multifractal spectra and singularity Eqs. (16) and (17) have the following properties:

$$
\begin{aligned}
& \alpha_{\max }=\alpha(-\infty)=-\frac{\log \left(d_{1} / m_{1}\right)}{\log h}, \\
& \alpha_{\min }=\alpha(\infty)=-\frac{\log \left(d_{2} / m_{2}\right)}{\log h}, \\
& \alpha(0)=-\frac{1}{\log h}\left[\frac{m_{1} \log \left(d_{1} / m_{1}\right)+m_{2} \log \left(d_{2} / m_{2}\right)}{m_{1}+m_{2}}\right],
\end{aligned}
$$

and

$$
\begin{aligned}
f\left(\alpha_{\max }\right) & =\frac{\log m_{1}}{\log h}, \\
f\left(\alpha_{\min }\right) & =\frac{\log m_{2}}{\log h}, \\
f(\alpha(0)) & =\frac{\log \left(m_{1}+m_{2}\right)}{\log h}, \\
\Delta f_{R} & =\frac{f(\alpha(0))}{f\left(\alpha_{\max }\right)}=\frac{\log \left(m_{1}+m_{2}\right)}{\log m_{1}}, \\
\Delta f_{L} & =\frac{f(\alpha(0))}{f\left(\alpha_{\min }\right)}=\frac{\log \left(m_{1}+m_{2}\right)}{\log m_{2}} .
\end{aligned}
$$

Therefore,

$$
\begin{aligned}
\Delta \alpha & =-\frac{\log \left(\frac{d_{1} / m_{1}}{d_{2} / m_{2}}\right)}{\log h}, \\
\Delta_{R} & =\frac{\Delta f_{l}}{\Delta f_{r}}=\frac{\log m_{2}}{\log m_{1}}, \\
R & =\frac{\Delta \alpha_{L}}{\Delta \alpha_{R}}=\frac{\alpha(0)-\alpha(\infty)}{\alpha(-\infty)-\alpha(0)}=\frac{m_{2}}{m_{1}}, \\
\Delta \tau & =\frac{d_{1} d_{2}}{\log h\left(d_{1}+d_{2}\right)^{2}}\left[\log \left(\frac{d_{1} / m_{1}}{d_{2} / m_{2}}\right)\right]^{2} .
\end{aligned}
$$

Figure $2 \mathrm{a}$ and $\mathrm{b}$ show the curves of $f(\alpha)$ and $\alpha$ generated by the binomial multiplicative cascade processes with different parameters for the partitions. It can be observed that the spectra are asymmetrical, and the asymmetry index is equal to the ratio of the two values $m_{2}$ and $m_{1}$ or $R=m_{2} / m_{1}$. The fractal dimensions at the two ends of the spectra are related to the ratio of the logarithmic transformation of values $\log \left(m_{1}\right) / \log h$ and $\log \left(m_{2}\right) / \log h$, which do not always reach zero; as a matter of fact, these values reach zero only if $m_{1}=1$ and $m_{2}=1$, respectively. The box-counting dimension (or the maximum value of the spectra) is related to the ratio of the logarithmic transformation of the sum of the two scales and the total segment $h, \log \left(m_{1}+m_{2}\right) / \log h \leq 1$; equality holds only if $m_{1}+m_{2}=h$. Both multifractality indices $(\Delta \alpha$ and $\Delta \tau)$ are proportional to the logarithmic transformation of $\left(d_{1} / m_{1}\right) /\left(d_{2} / m_{2}\right)$. Thus far, the multifractal indices are explicitly related to the parameters involved in the multiplicative cascade processes. Generally speaking, the larger the values of $\alpha(0), R$, and $\Delta \alpha$ or $\Delta \tau$, the more complex the distribution, which has the consequences of stronger multifractality, additional variability of the high values of measure $\mu$ with positive singularity $\alpha<\alpha(0)$ and spectra that bend towards the right. These parameters can be used to mimic the asymmetrical multifractal distribution that could have been generated by the binomial multiplicative cascade processes.

The new model proposed in this paper is a general model in comparison with the $p$ model, Koscielny-Bunde's model and Macek's model. For example, letting $d_{1}=p$ and $d_{2}=$ $1-p$, and $m_{1} / h=m_{2} / h=0.5$, the five-parameter model 

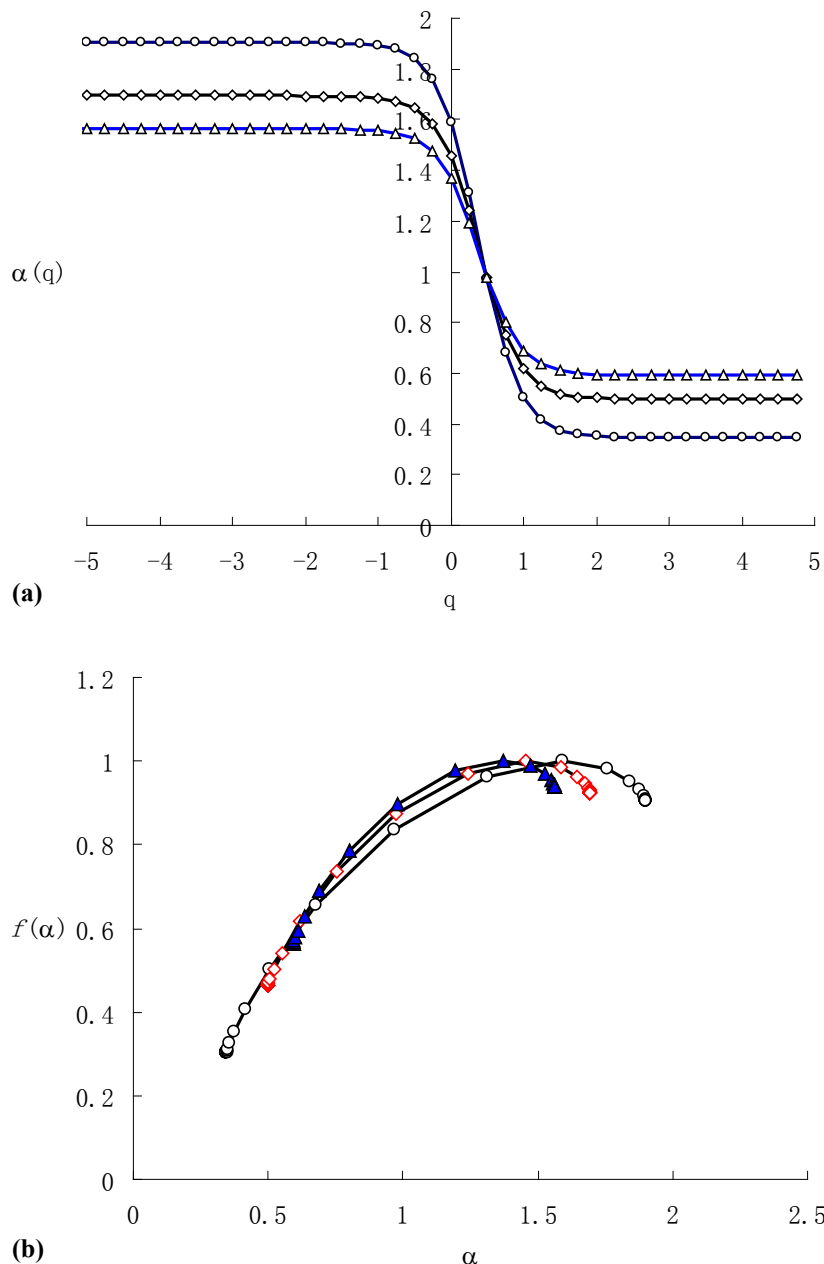

(b)

$\alpha$

Fig. 2. (a) Singularity functions $\alpha(q)$ obtained from asymmetrical de Wijs model values of $d_{1}=0.1$ and $d_{2}=0.9$ and different partitions $m_{1}=2, m_{2}=6$, and $h=8$ (dots), $m_{1}=4, m_{2}=12, h=16$ (diamonds) and $m_{1}=8, m_{2}=24$, and $h=32$ (triangles), respectively. Ranges of $q$ value are similar for all models. (b) The results of spectra function $f(\alpha)$ for different partitions with value $d_{1}=0.1$ and $d_{2}=0.9$.

reduces to the standard $p$ model (Meneveau and Sreenivasan, 1987), which gives symmetrical multifractal spectra with $f\left(\alpha_{\max }\right)=0$ and $f\left(\alpha_{\min }\right)=0$. Koscielny-Bunde's two-parameter model (Koscielny-Bunde et al., 2006) corresponds to a special case of the five-parameter model if letting $d_{1}=a$ and $d_{2}=b, d_{1}+d_{2} \geq 1$ and $m_{1} / h=m_{2} / h=1 / 2$. The model only involves two parameters, $a$ and $b$, and $\Delta \alpha=$ $\log (a / b) / \log 2$ and $R=1$, and gives symmetrical multifractal spectra with $f\left(\alpha_{\max }\right)=0$ and $f\left(\alpha_{\min }\right)=0$. Similarly, Macek's three-parameter model (Macek, 2007) corresponds to a special case of the five-parameter model if letting $d_{1}=p$ and $d_{2}=1-p$, and $m_{1} / h=l_{1}$ and $m_{2} / h=l_{2}$. The model involves three parameters $p, l_{1}$, and $l_{2}$. The five-parameter model can not only describe asymmetrical multifractal spectra but also gives explicit relationships between the multi- fractal indices and the parameters involved in the binomial multiplicative cascade processes. Moreover, the new model is able to generate asymmetrical multifractal distributions with nonzero fractal dimensions of the extreme values at two ends of the spectrum, i.e., $f\left(\alpha_{\max }\right) \neq 0$ and $f\left(\alpha_{\min }\right) \neq 0$. In the 2-D cascade model with anisotropic partition processes (Cheng, 2005), instead of the same values of $d_{1}$ and $d_{2}$ for $m_{1}$ and $m_{2}$ subareas in the partition, it uses different values of $d_{i}$, $i=1, \ldots, h$, among subareas. Therefore, the five-parameter model proposed in the current paper is a special case of the 2-D anisotropic cascade model (Cheng, 2005).

\section{Procedure of the general five-parameter model for mimicking the binomial asymmetrical multifractal distribution}

It has been shown that the multiplicative cascade model with the five parameters $d_{1}, d_{2}, m_{1}, m_{2}$ and $h$ can not only generate asymmetrical multifractal distributions but also the multifractal indices commonly used in the literature that can be explicitly expressed as a function of the five parameters. Therefore, these indices, i.e., multifractality and asymmetry, can be used to characterize the asymmetrical binomial multifractal distribution and the fractal dimension spectra. The procedures are described as follows.

\subsection{Step 1}

The values of $m_{1}, m_{2}$ and $h$ can be estimated using the following three characteristic points of the fractal dimension spectra:

$$
\begin{gathered}
m_{1}=e^{f\left(\alpha_{\max }\right) \log h}, \\
m_{2}=e^{f\left(\alpha_{\min }\right) \log h}, \\
h=e^{\frac{\log \left(m_{1}+m_{2}\right)}{f(\alpha(0))}} .
\end{gathered}
$$

With the values of maximum fractal dimension $f(\alpha(0))$ and the dimensions at the two ends of the spectra $f\left(\alpha_{\max }\right)$ and $f\left(\alpha_{\min }\right)$, we can form iterations about $h$ as the objective function of input $h$, which can be easily implemented in Microsoft Excel. For each iteration, an input value of $h$ will yield an output value of $h$. The input value of $h$ with the newly calculated value of $h$ can be compared for decisions with respect to the termination of the iteration processes. An adjusted value of $h$, e.g., the average of the estimated and the input values of $h$, can be used as the new input of $h$ for the next step of the iteration. The iteration processes halt when the input value of $h$ is close to the output values of $h$. Next, the other two values of $m_{1}$ and $m_{2}$ are calculated accordingly.

\subsection{Step 2}

After the values of all three parameters $m_{1}, m_{2}$ and $h$ are estimated, the following relationships are used to determine 
the values of $d_{1}$ and $d_{2}$ :

$d_{1}=e^{-m_{1} \alpha_{\max } \log h}$,

$d_{2}=e^{-m_{2} \alpha_{\min } \log h}$.

To illustrate the application of the five-parameter binomial multiplicative cascade model, in the next section we introduce several examples. The fractal dimension functions calculated and published in the literature are reanalyzed using the five-parameter model.

\section{Application examples}

\subsection{Example 1: de Wijs's zinc values}

De Wijs (1951) studied assay values from the Pulacayo sphalerite-quartz vein in Bolivia. These data have been analyzed using multifractal modeling and spatial analysis (Cheng and Agterberg, 1996). It has been shown that these data can be approximated using a binomial multiplicative cascade model. The results of Cheng and Agterberg (1996) will be further analyzed in this work by means of the fiveparameter binomial multiplicative cascade model. Cheng (1999b) applied a gliding-box multifractal method to calculate the fractal dimension spectra of the distribution of de Wijs's zinc values (Fig. 3a). The range of order of moment $q$ was between -20 and 20 . The estimated values of $f(\alpha)$ are given as follows:

$$
\begin{aligned}
\alpha_{\max } & =1.30, \alpha_{\min }=0.75, \quad f(\alpha(0))=1.00 \\
f\left(\alpha_{\max }\right) & =0, \quad f\left(\alpha_{\min }\right)=0.13, \quad \alpha(0)=1.013 .
\end{aligned}
$$

According to the iteration method introduced in the previous section, we can estimate the values of five parameters as

$h=2.1, \quad m_{1}=1, \quad m_{2}=1.1$

$d_{1}=0.38, d_{2}=0.63$.

Therefore,

$\Delta \alpha=0.75, \quad \Delta \tau=0.033$ and $R=1.1$.

The curves of $f(\alpha)$ based on the model with these five values are superimposed on the curve of $f(\alpha)$ estimated by the gliding-box multifractal method (see Fig. 3a). The results indicate that the distribution is multifractal and is close to symmetrical.

\subsection{Example 2: Szczepaniak and Macek's solar wind data}

Szczepaniak and Macek (2008) modeled solar wind turbulence using an asymmetrical multiplicative cascade model based on a two-scale weighted cantor set with experimental data from solar wind measured in situ by the Advanced Composition Explorer spacecraft during the solar maximum
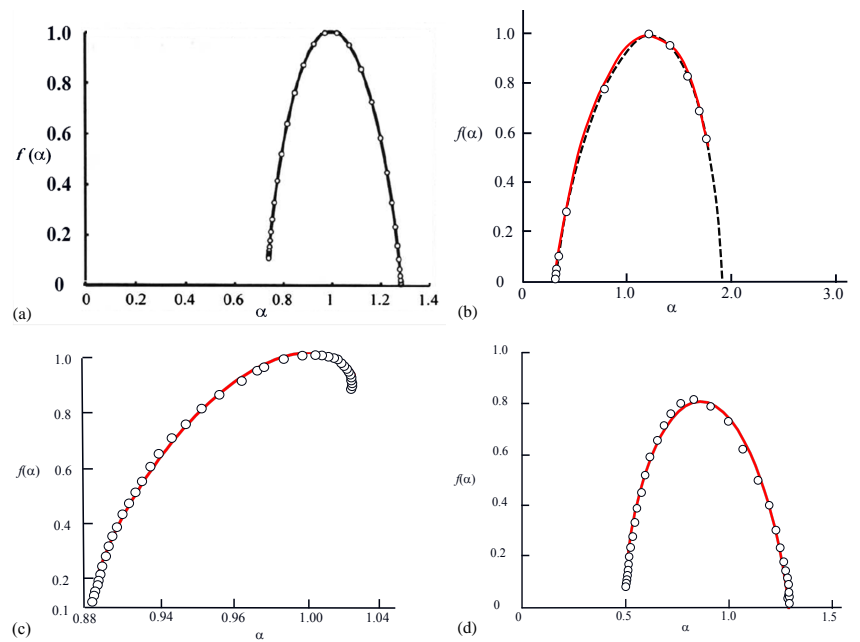

Fig. 3. Results showing fractal dimension functions (dots) calculated by previous authors published in literature and fitted by the five-parameter binomial multiplicative cascade model proposed in the current paper (solid line). (a) Fractal dimension spectrum calculated for de Wijs's zinc values by means of gliding-box method (Cheng, 1999b) and binomial multiplicative cascade model with $m_{1}=1, m_{2}=1.1, h=2.1, d_{1}=0.38$, and $d_{2}=0.63$. (b) Fractal dimension spectrum calculated for the energy transfer rate in the solar wind turbulence at solar minimum (2006) fitted by the model proposed by Szczepaniak and Macek (2008) (dashed line). The red solid line fitted by the five-parameter binomial multiplicative cascade model with $m_{1}=1, m_{2}=1.74, h=2.74, d_{1}=0.738$, and $d_{2}=0.292$. (c) Fractal dimension spectrum calculated for time series of synthetic earthquakes by Muñoz-Diosdado et al. (2004) and binomial multiplicative cascade model with $m_{1}=7.17, m_{2}=1.54$, $h=8.71, d_{1}=0.779$, and $d_{2}=0.226$. (d) Fractal dimension spectrum calculated for human posture data by wavelet transform modulus multifractal method (WTMM) (Shimizu et al., 2002) and binomial multiplicative cascade model with $m_{1}=1.219, m_{2}=1.00$, $h=2.69, d_{1}=0.728$, and $d_{2}=0.276$.

(2001) and minimum (2006) at 1 AU. The results obtained show the asymmetrical multifractal nature of the different states of the solar wind. For comparison purposes, the fractal dimension spectra for the solar minimum (2006) at $1 \mathrm{AU}$ are fitted using the five-parameter binomial multiplicative cascade model. Based on the visual determination from the fractal dimension spectrum of solar minimum (2006) in Fig. 3b, the characteristic values of $f(\alpha)$ are estimated as follows:

$$
\begin{aligned}
\alpha_{\max } & =1.77, \quad \alpha_{\min }=0.30, \quad f(\alpha(0))=1.00 \\
f\left(\alpha_{\max }\right) & =0.55, \quad f\left(\alpha_{\min }\right)=0.00 .
\end{aligned}
$$

Accordingly, the values of the five parameters are estimated as follows:

$$
\begin{aligned}
h & =2.74, \quad m_{1}=1, \quad m_{2}=1.74 \\
d_{1} & =0.738, \quad d_{2}=0.292 .
\end{aligned}
$$


Therefore,

$\Delta \alpha=1.469, \quad \Delta \tau=0.198$ and $R=1.74$.

The curve fitted by the model with the five values above is superimposed on the results obtained by Szczepaniak and Macek (2008) (see Fig. 3b). It can be observed that the model fits the data well. The values estimated for multifractality and symmetry are slightly different from those obtained by Szczepaniak and Macek (2008). The discrepancy is primarily due to the differences in the fractal dimensions at the right end of the spectra. It is noteworthy that the model that Szczepaniak and Macek (2008) used to fit the data with the curve reaches zero at both ends, whereas the curve fitted by the new model reaches zero only at one end but not at the other end. The results also indicate that the distribution has strong multifractality and a left-skewed asymmetry. It should be mentioned that the spectra curve shown in Fig. 3b was estimated with the $q$ value ranging from -2 to 8 . This indicates that the values of $\alpha_{\min }$ and $f\left(\alpha_{\min }\right)$ on the left end of $f(\alpha)$ are estimated with less uncertainty, whereas the values on the right could involve large uncertainty due to a small range of negative $q$ value.

\subsection{Example 3: time series of synthetic earthquakes (Muñoz-Diosdado et al., 2004)}

Muñoz-Diosdado et al. (2004) modeled the time series of synthetic earthquakes using multifractal distribution with theoretical spectra generated via multiplicative processes. This group found that the time series of synthetic earthquakes depicts multifractality, and the multifractal spectra can be asymmetrical. The asymmetrical multifractal distribution was calculated with high accuracy by Muñoz-Diosdado et al. (2004) with the $q$ value ranging from -30 to 30 . The results were fitted using the five-parameter model. From visual determination of the fractal dimension spectra in Fig. 3c, the characteristic values of $f(\alpha)$ are estimated as follows:

$$
\begin{aligned}
\alpha_{\max } & =1.024, \quad \alpha_{\min }=0.886, \quad f(\alpha(0))=1.00 \\
f\left(\alpha_{\max }\right) & =0.9, \quad f\left(\alpha_{\min }\right)=0.2 .
\end{aligned}
$$

Accordingly, the values of five parameters are estimated as follows:

$$
\begin{aligned}
h & =8.71, \quad m_{1}=7.17, \quad m_{2}=1.54 \\
d_{1} & =0.779, \quad d_{2}=0.226 .
\end{aligned}
$$

Therefore,

$$
\Delta \alpha=0.133, \quad \Delta \tau=0.003 \text { and } R=0.214 .
$$

The results show these data depict weak multifractality with a strong right-skewed asymmetry.

\subsection{Example 4: multifractal analysis of human posture data by Shimizu et al. (2002)}

Shimizu et al. (2002) analyzed human posture data using a wavelet transform modulus multifractal method (WTMM) (Muzy et al., 1991). This group proposed a vector of three multifractal indices as a measure of the complexity in human standing data. Figure $3 \mathrm{~d}$ shows an example of a fractal spectrum obtained by Shimizu et al. (2002, Fig. 4d) from real standing data from a randomly selected healthy subject. The estimations of $f(\alpha)$ were calculated with the $q$ value ranging from -10 to 10 . Based on visual determination of the fractal dimension spectra in Fig. 3d, the characteristic values of $f(\alpha)$ are estimated as follows:

$$
\begin{aligned}
\alpha_{\max } & =0.52, \alpha_{\min }=1.28, \quad f(\alpha(0))=0.805 \\
f\left(\alpha_{\max }\right) & =0, \quad f\left(\alpha_{\min }\right)=0.2 .
\end{aligned}
$$

Accordingly, the values of five parameters are estimated as follows:

$$
\begin{aligned}
h & =2.69, \quad m_{1}=1, \quad m_{2}=1.219 \\
d_{1} & =0.728, \quad d_{2}=0.281 .
\end{aligned}
$$

Therefore,

$\Delta \alpha=0.76, \Delta \tau=0.118$ and $R=1.219$.

The results show the data depict strong multifractality and a left-skewed asymmetry. The maximum fractal dimension of the spectra is $0.805(<1)$, which corresponds to $m_{1}+m_{2}=$ $2.219<2.69$.

\section{Conclusions and discussion}

It has been demonstrated that the five-parameter binomial multiplicative cascade model proposed in the current paper can be used to simulate the generation of an asymmetrical multifractal distribution. In addition to the explicit relationships found between the parameters involved in the binomial multiplicative cascade processes, the commonly used multifractal indices, i.e., multifractality, asymmetry, and fractal dimensions of high and low values, provide insight into the physical meaning of multifractal indices, which are intuitive for the use of multifractal modeling of real-world data. The theoretical discussions were followed by case studies with published data in the literature that have also shown that the five-parameter model is superior to other models such as Macek's model for the following two reasons: the new model provides explicit relationships between the parameters involved in the binomial multiplicative cascade processes and multifractal indices, and more importantly, the new model is able to generate more general asymmetrical multifractal distributions, e.g., nonzero fractal dimensions of the high and low values, $f\left(\alpha_{\max }\right)>0$ and $f\left(\alpha_{\min }\right)>0$. It should be noted 
that although the binomial multiplicative cascade processes can generate asymmetrical multifractal distributions, certain multifractal distributions are due to other complex processes for which binomial multifractal models may not be applicable. The other weakness of the general binomial model is that because the five parameters are estimated based on a multifractal distribution estimated using other types of methods, the accuracy of the parameters may be affected by the estimated multifractal distribution due to various factors including FSE bias and mixing trend in the real data. Nevertheless, the model proposed in this paper is applicable to simple multifractal distributions commonly estimated from geoscience data sets. The explicit relationships between the multiplicative cascade processes and the basic multifractal indices are useful for understanding the physical meaning of multifractal indices although the multifractal distribution might not be generated by binomial cascade processes. However, other properties of the generalized binomial multifractal distribution such as spatiotemporal stationarity, lacunarity and variability remain for further investigation.

Acknowledgements. Thanks are due to Francesco Serinaldi and another reviewer for their critical review and constructive comments. This research has been jointly supported by the research project: Quantitative models for prediction of strategic mineral resources in China (201211022), by the China Geological Survey; and by NSERC Discovery Research: Research and development of multifractal methods and GIS technology for mineral exploration and environmental assessments (ERC-OGP0183993). The results of the study were partly presented at the conferences of the EGU in 2010 and the 35 th IGC in 2012.

Edited by: L. Telesca

Reviewed by: F. Serinaldi and one anonymous referee

\section{References}

Agterberg, F. P.: Multifractal modeling of the sizes and grades of giant and supergiant deposits, Int. Geol. Rev., 37, 1-8, 1995.

Agterberg, F. P.: Multifractal simulation of geochemical map patterns, in: Geologic modeling and simulation: Sedimentary Systems, edited by: Merriam, D. F. and Davis, J. C., Kluwer, New York, 327-346, 2001.

Agterberg, F. P.: New applications of the model of de Wijs in regional geochemistry, Math. Geol., 39, 1-26, 2007 a.

Agterberg, F. P.: Mixtures of multiplicative cascade models in geochemistry, Nonlin. Processes Geophys., 14, 201-209, doi:10.5194/npg-14-201-2007, 2007b.

Cheng, Q.: Discrete multifractals, Math. Geol., 29, 245-266, 1997a.

Cheng, Q.: Multifractal modelling and lacunarity analysis, Math. Geol., 29, 919-932, 1997b.

Cheng, Q.: Multifractality and spatial statistics, Comput. Geosci., 25, 949-961, 1999a.

Cheng, Q.: The gliding box method for multifractal modeling, Comput. Geosci., 25, 1073-1079, 1999b.
Cheng, Q.: Multifractal distribution of eigenvalues and eigenvectors from 2D multiplicative cascade multifractal fields, Math. Geol., 37, 915-927, 2005.

Cheng, Q.: Multifractal imaging filtering and decomposition methods in space, Fourier frequency, and eigen domains, Nonlin. Processes Geophys., 14, 293-303, doi:10.5194/npg-14-293-2007, 2007b.

Cheng, Q.: Mapping singularities with stream sediment geochemical data for prediction of undiscovered mineral deposits in Gejiu, Yunnan Province, China, Ore Geol. Rev., 32, 314-324, 2007 a.

Cheng, Q.: Non-linear theory and power-law models for information integration and mineral resources quantitative assessments, Math. Geosci., 40, 503-532, 2008.

Cheng, Q.: Multiplicative cascade processes and information integration for predictive mapping, Nonlin. Processes Geophys., 19, 57-68, doi:10.5194/npg-19-57-2012, 2012.

Cheng, Q. and Agterberg, F. P.: Multifractal modeling and spatial statistics, Math. Geol., 28, 1-16, 1996.

Cheng, Q. and Agterberg, F. P.: Singularity analysis of ore-mineral and toxic trace elements in stream sediments, Comput. Geosci., 35, 234-244, 2009.

Cheng, Q., Agterberg, F. P., and Ballantyne, S. B.: The separation of geochemical anomalies from background by fractal methods, J. Geochem. Explor., 51, 109-130, 1994.

Cheng, Q., Li, L., and Wang, L.: Characterization of peak flow events with local singularity method, Nonlin. Processes Geophys., 16, 503-513, doi:10.5194/npg-16-503-2009, 2009.

de Wijs, H. J.: Statistics of ore distribution, Part 1: Frequency distribution of assay values, Geol. Mijnbouw, 13, 365-375, 1951.

Feder, J.: Fractals, Plenum Press, New York, 283 pp., 1988.

Frisch, U., Sulem, P. L., and Nelkin, M.: A simple dynamical model of intermittency in fully develop turbulence, J. Fluid Mech., 87, 719-724, 1978.

Grech, D. and Pamuła, G.: Multifractal background noise of monofractal signals, Acta Phys. Pol. A, 121, B34-39, 2012.

Grech, D. and Pamuła, G.: On the multifractal effects generated by monofractal signals, Physica A, 392, 5845-5864, 2013.

Gu, G. F and Zhou, W. X.: Detrended fluctuation analysis for fractals and multifractals in higher dimensions, Phys. Rev. E, 74, 061104, doi:10.1103/PhysRevE.74.061104, 2006.

Gupta, V. K. and Waymire, E. C.: A statistical analysis of mesoscale rainfall as a random cascade, J. Appl. Meteorol., 32, 251-267, 1993.

Halsey, T. C., Jensen, M. H., Kadanoff, L. P., Procaccia, I., and Shraiman, B.: Fractal measures and their singularities: the characterization of strange sets, Phys. Rev. A, 33, 1141-1151, 1986.

Kantelhardt, J. W., Zschiegner, S. A., Koscielny-Bunde, E., Havlin, S., Bunde, A., and Stanley, H. E.: Multifractal detrended fluctuation analysis of nonstationary time series, Physica A, 316, 87114, 2002.

Koscielny-Bunde, E., Kantelhardt, J. W., Braund, P., Bunde, A, and Havlin, S.: Long-term persistence and multifractality of river runoff records: Detrended function studies, J. Hydrol., 322, 120 137, 2006.

Lombardo, F., Volpi, E., and Koutsoyiannis, D.: Rainfall downscaling in time: theoretical and empirical comparison between multifractal and Hurst-Kolmogorov discrete random cascades, Hydrolog. Sci. J., 57, 1052-1066, 2012. 
Lombardo, F., Volpi, E., Koutsoyiannis, D., and Papalexiou, S. M.: Just two moments! A cautionary note against use of high-order moments in multifractal models in hydrology, Hydrol. Earth Syst. Sci. Discuss., 10, 4627-4654, doi:10.5194/hessd-10-46272013, 2013.

Lovejoy, S. and Schertzer, D.: Scaling and multifractal fields in the solid earth and topography, Nonlin. Processes Geophys., 14, 465-502, doi:10.5194/npg-14-465-2007, 2007.

Macek, W. M.: Multifractality and intermittency in the solar wind, Nonlin. Processes Geophys., 14, 695-700, doi:10.5194/npg-14695-2007, 2007.

Macek, W. M. and Szczepaniak, A.: Generalized two-scale weighted Cantor set model for solar wind turbulence, Geophys. Res. Lett., 35, L02108, doi:10.1029/2007GL032263, 2008.

Macek, W. M. and Wawrzaszek, A.: Multifractal two-scale Cantor set model for slow solar wind turbulence in the outer heliosphere during solar maximum, Nonlin. Processes Geophys., 18, 287294, doi:10.5194/npg-18-287-2011, 2011.

Malamud, B. D., Turcotte, D. L., and Barton, C. C.:, The 1993 Mississippi river flood: a one hundred or a one thousand year event?, Environ. Eng. Geosci., 2, 479-486, 1996.

Malamud, B. D., Turcotte, D. L., Guzzetti, F., and Reichenbach, P.: Landslide inventories and their statistical properties, Earth Surf. Proc. Land., 29, 687-711, 2004.

Mandelbrot, B. B.: The Fractal Geometry in Nature, Henry Holt and Company, 468 pp., 1983.

Mandelbrot, B. B.: Multifractal measures, especially for the geophysicist, Pure Appl. Geophys., 131, 5-42, 1989.

Menabde, M. and Sivapalan, M.: Modeling of rainfall time series and extremes using bounded random cascades and Levy-stable distributions, Water Resour. Res., 36, 3293-3300, 2000.

Meneveau, C. and Sreenivasan, K. R.: Simple multifractal cascade model for fully developed turbulence, Phys. Rev. Lett., 59, 14241427, 1987.

Muñoz-Diosdado, A., Angulo-Brown, F., and del RÍO-Correa, J. L.: Multifractal analysis of a spring-block seismic fault, in: Proceedings of 13th World Conference on Earthquake Engineering, Vancouver, B.C., Canada, 1-6 August 2004, Paper No. 525, 2004.

Muzy, J. F., Bacry, E., and Arneodo, A.: Wavelets and multifractal formalism for singular signals: application to turbulences data, Phys. Rev. Lett., 67, 3515-3518, 1991.

Over, T. M. and Gupta, V. K.: A space-time theory of mesoscale rainfall using random cascades, J. Geophys. Res., 101, 2631926331, 1996.

Plotnick, R. E., Gardner, R. H., and O’Neill, R. V.: Lacunarity indices as measures of landscape texture, Landscape Ecol., 8, 201$211,1993$.
Rosas, A., Nogueira Jr., E., and Fontanari, J. F.: Multifractal analysis of DNA walks and trails, Phys. Rev. E, 66, 061906-1-0619066, 2002.

Schertzer, D. and Lovejoy, S.: The dimension and intermittency of atmospheric dynamics - Multifractal cascade dynamics and turbulent intermittency, in: Turbulent Shear Flow, edited by: Launder, B., Springer-Verlag, New York, 7-33, 1985.

Schertzer, D. and Lovejoy, S.: Physical modeling and analysis of rain and clouds by anisotropic scaling of multiplicative processes, J. Geophys. Res., 92, 9693-9714, 1987.

Schertzer, D., Lovejoy, S., Schmitt, F., Chigirinskaya, Y., and Marsan, D.: Multifractal cascade dynamics and turbulent intermittency, Fractals, 5, 427-471, 1997.

Serinaldi, F.: Multifractality, imperfect scaling and hydrological properties of rainfall time series simulated by continuous universal multifractal and discrete random cascade models, Nonlin. Processes Geophys., 17, 697-714, doi:10.5194/npg-17-6972010, 2010.

Shimizu, Y., Thurner, S., and Ehrenberger, K.: Multifractal spectra as a measure of complexity in human posture, Fractals, 10, 103116, 2002.

Sornette, D.: Critical Phenomena in Natural Sciences: Chaos, Fractals, Selforganization and Disorder, 2nd Edn., Springer Series in Synergetics, Springer, New York, 2004.

Szczepaniak, A. and Macek, W. M.: Asymmetric multifractal model for solar wind intermittent turbulence, Nonlin. Processes Geophys., 15, 615-620, doi:10.5194/npg-15-615-2008, 2008.

Telesca, L. and Lovallo, M.: Revealing competitive behaviors in music by means of the multifractal detrended fluctuation analysis: application to Bach's Sinfonias, P. Roy. Soc. A-Math. Phy., 467, 3022-3032, 2011.

Telesca, L., Lovallo, M., Lepenna, V., and Macchiato, M.: Longrange correlations in two-dimension spatio-temporal seismic fluctuations, Physica A, 377, 279-284, 2007.

Turcotte, D. L.: Fractals and Chaos in Geology and Geophysics, 2nd Edn., Cambridge University Press, 1997.

Veneziano, D.: Multifractality of rainfall and scaling of intensityduration-frequency curves, Water Resour. Res., 38, 1-12, 2002.

Xie, S. and Bao, Z.: Fractal and multifractal Properties of Geochemical Fields, Math. Geol., 36, 847-864, 2004.

Yaglom, A. M.: The influence of the fluctuation in energy dissipation on the shape of turbulent characteristics in the inertial interval, Sov. Phys. Dokl. (English Translation), 2, 26-30 1966. 